\title{
A Berry Picking Robot With A Hybrid Soft-Rigid Arm: Design and Task Space Control
}

\author{
Naveen Kumar Uppalapati*, Benjamin T Walt ${ }^{\dagger}$, Aaron J Havens ${ }^{\ddagger}$, Armeen Mahdian ${ }^{\S}$, Girish Chowdhary $₫$ \\ and Girish Krishnan* \\ ${ }^{*}$ Industrial and Enterprise Systems Engineering, ${ }^{\dagger}$ Mechanical Science and Engineering, ${ }^{\ddagger}$ Aerospace Engineering, \\ ${ }^{\S}$ Electrical and Computer Engineering, ${ }^{\Uparrow}$ Agricultural and Biological Engineering, \\ University of Illinois at Urbana-Champaign, Urbana, IL 61801 \\ Email: \{uppalap2, walt, ahavens2, amahdia2, girishc and gkrishna\} @illinois.edu
}

\begin{abstract}
We present a hybrid rigid-soft arm and manipulator for performing tasks requiring dexterity and reach in cluttered environments. Our system combines the benefit of the dexterity of a variable length soft manipulator and the rigid support capability of a hard arm. The hard arm positions the extendable soft manipulator close to the target, and the soft arm manipulator navigates the last few centimeters to reach and grab the target. A novel magnetic sensor and reinforcement learning based control is developed for end effector position control of the robot. A compliant gripper with an IR reflectance sensing system is designed, and a k-nearest neighbor classifier is used to detect target engagement. The system is evaluated in several challenging berry picking scenarios.
\end{abstract}

\section{INTRODUCTION}

Robots capable of dexterous manipulation in cluttered environments can significantly impact many applications. For example, shortage of qualified human labor is a key challenge facing US farmers [23, 15], leading to smaller profit margins, and preventing the adoption of truly sustainable agricultural practices [4, 12, 13]. The labor shortage critically affects berry farms and orchards, because tasks such as picking berries or pruning branches require significant dexterity. Simple automation approaches that are popular in row-crops such as corn and soybean [3], do not work well in more complicated perennial crop environment. In addition, traditional industrial "hard" robot arms have been difficult to adopt for messy, cluttered, and delicate plants [11]. It is believed that the emerging field of pneumatically actuated soft robotics [22, 17] could be the answer for implementing robotic automation in challenging applications such as berry picking [5]. However, soft-robot arms on their own have several challenges. The primary one being low to moderate actuation powers available in a mobile, untethered environment, coupled with the fact that material flexibility of the robot arm significantly limits payload capacity. Furthermore, payloads on these arms can warp the workspace, making the control problem harder, and the warping can worsen with increasing length thus affecting accuracy and precision [24].

In this work, we present a new hybrid soft-rigid arm concept system for applications requiring dexterity and reach. We term this system SoftAgbot since our primary application is picking berries in greenhouses, although SoftAgbot can be applied to many applications beyond agriculture. Our concept system

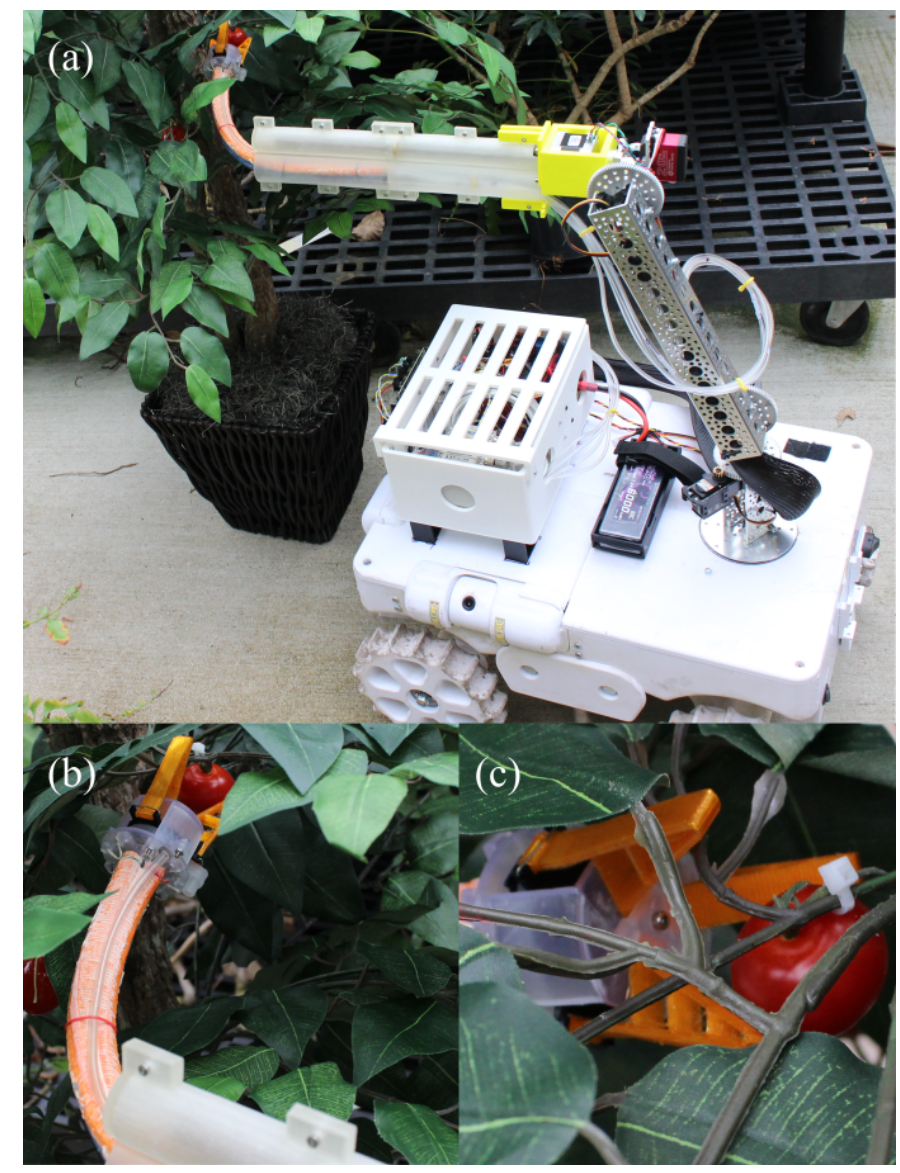

Fig. 1. (a) SoftAgbot system (mounted on a TerraSentia mobile rover) presented in this work picking a berry, (b) and (c) ability to reach bush interiors using a flexible soft continuum arm.

combines the benefits of the dexterity of a soft manipulator with the rigid support capability of a hard arm. The idea is that the hard arm positions the extendable soft manipulator close to the target, and the soft arm manipulator navigates the last few centimeters $(25 \mathrm{~cm}$ in the presented case) to reach and grab the target.

\section{A. Contributions and their significance}

The main contributions of our work are: 

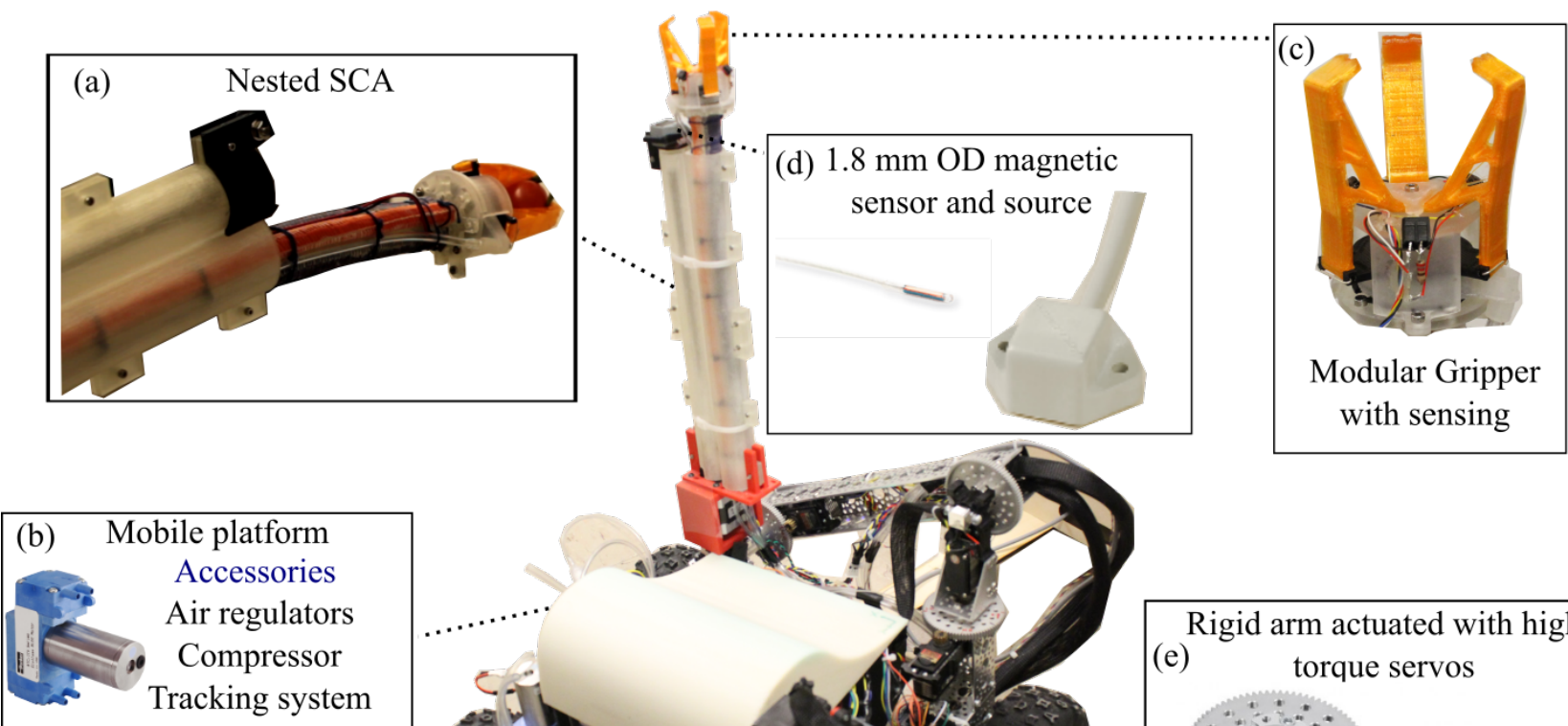

Controllers

Rasberry Pi

RoboClaw

NI myRIO
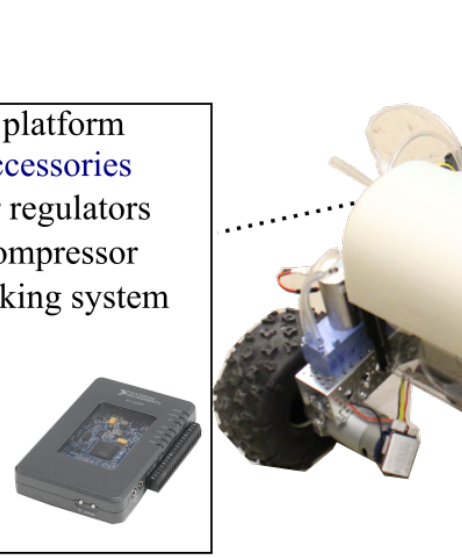

$40 \mathrm{~mm}$

(d) $1.8 \mathrm{~mm}$ OD magnetic sensor and source
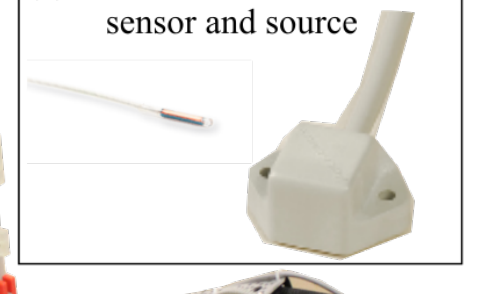

Fig. 2. SoftAgbot system prototype showing different subsystems and components implemented on a smaller robot.

1) We present a novel hybrid soft-hard arm system with a compliant gripping end effector that is capable of reaching and manipulating soft objects at significant distances from the robot.

2) The presented system is completely self-contained and has been mounted on two robots, one of which is the commercially available TerraSentia mobile rover (see Figure 1], [16]).

3) We utilize a novel magnetic sensor and reinforcement learning based control for end effector position control of the robot.

4) We employ IR reflectance sensors mounted on the gripper and a k-nearest neighbor classifier to predict the success of a gripping action to better operate in occluded environments.

5) We demonstrate that SoftAgbots have an unprecedented ability to reach difficult targets and apply a wide range of forces to achieve objectives that are very hard or nearly impossible for traditional "rigid only" armed robots.

The significance of our contributions is in presenting a pathway to creating robots capable of having the reach and dexterity of soft robots without the drawbacks of lack of strength and excessive workspace warping. In addition, our robot can be position controlled in the task space either using a set of end effector desired positions or a joystick that a human can use to control the position. This significantly simplifies the control of this pneumatically actuated soft robot, which would otherwise require manipulating a number of valves. Note that the SoftAgbot presented here is specific for berry harvesting, but the same system, with modifications can also be used to control weeds, detect insects and diseases by moving a camera throughout the dense plant canopy, and prune or thin branches.

\section{SYSTEM OVERVIEW}

The robot design is guided by the need to maximize dexterity, adaptability and safety while also increasing accuracy, and load bearing ability. The robot consists of a mobile platform with a three-link rigid manipulator. The third link has the capability to deploy a Variable Length Nested Soft (VaLeNS) arm [29] that can be extruded in and out of the link. The end of the soft arm has a compliant gripping end effector. The details of the design are presented below.

\section{A. Robot arm and degrees of freedom}

The robot arm is a three-link rigid arm as shown in Figure 3 The arm sits on a rotating base $\left(\theta_{1}\right)$ and has two revolute joints $\left(\theta_{2}\right.$ and $\left.\theta_{3}\right)$. The third link is capable of axial rotation $\left(\theta_{4}\right)$.

1) VaLeNS arm: The third link is a hollow 3D-printed shell that houses a compliant soft arm. The soft arm can extrude out of the shell to provide additional dexterity, reachability, 


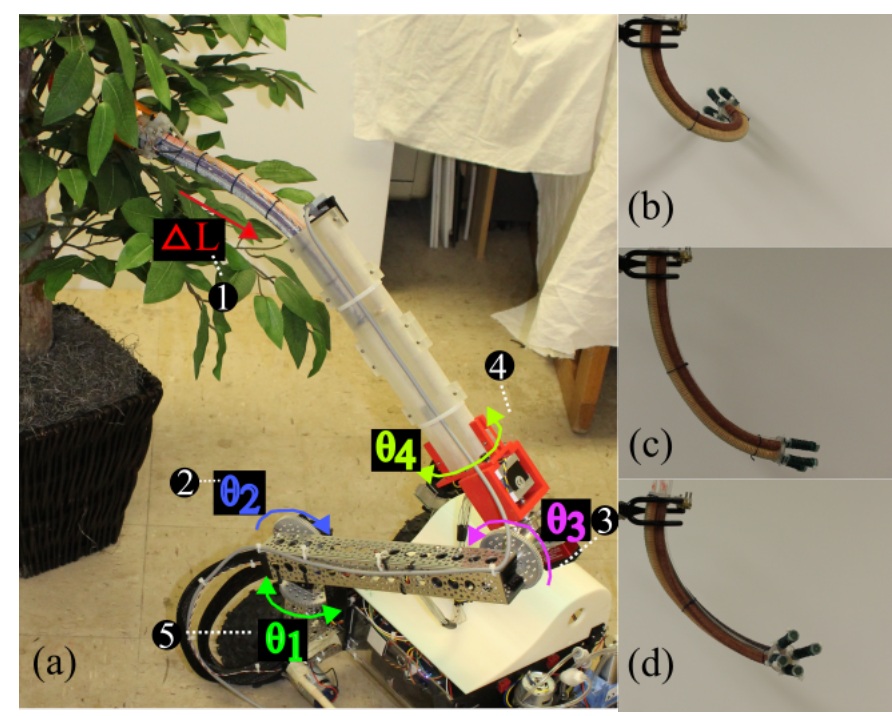

Fig. 3. Degrees of freedom of the robot (a) the rigid arm has three $\left(\theta_{1}\right.$ to $\left.\theta_{3}\right)$ d.o.f and the hybrid rigid soft arm has five $\left(\theta_{4}, \Delta L, \Delta \mathrm{B}, \Delta \mathrm{R}_{1}\right.$ and $\left.\Delta \mathrm{R}_{2}\right)$. The deformation of $\mathrm{BR}^{2}$ soft continuum arm with (b) $\Delta \mathrm{B}, \Delta \mathrm{R}_{1}$, (c) $\Delta \mathrm{B}$ and (d) $\Delta \mathrm{B}, \Delta \mathrm{R}_{2}$ actuation respectively.

and compliance. The 3D-printed shell encloses a NEMA 17size hybrid bipolar stepper motor that has an integrated 28 $\mathrm{cm}$ threaded rod as its output shaft (Pololu). This arrangement converts the motor into a linear actuator to extrude the soft arm in and out of the shell. Such a configuration is known as the VaLeNS arm and its workspace and dexterity were studied in [29].

2) Soft Continuum Arm: The $\mathrm{BR}^{2}$ Soft Continuum Arm (SCA) consists of a parallel combination of three pneumatically actuated Fiber Reinforced Elastomeric Enclosures (FREEs) [30, 24] that can bend $(B)$, and rotate in clockwise and counterclockwise directions $\left(R^{2}\right)$ respectively. A combination of spatial bending and axial twist or rotation results in a spiral deformation mode [28], which yields a large spatial workspace and dexterity (its deformation modes are shown in Figure 3(b)-(d)) with a compact design. Furthermore, the $\mathrm{BR}^{2}$ design can achieve variable stiffness in its pure bending configuration by differential pressurization of the bending and rotating FREEs.

Thus the three link rigid arm with the appended VaLeNS arm has a total of eight actuated degrees of freedom: $\theta_{1}-\theta_{4}$, $\Delta L, B$ (bending of the soft arm), and $R^{2}$ (clockwise and counterclockwise rotation of the arm). The ensuing dexterity enables the arm to approach a plant precisely and deploy the dexterous SCA as needed to explore the internal regions of the plant.

\section{B. Mobile platform}

The robot arm is mounted on a mobile platform as shown in Figure 2. The mobile platform encompasses a set of accessories and controllers to control the wheels, three-link rigid arm and the VaLeNS arm. We use a National Instruments myRIO to send the commands to the four pressure regulator valves (SMC, ITV0031-2UBL), RoboClaw motor

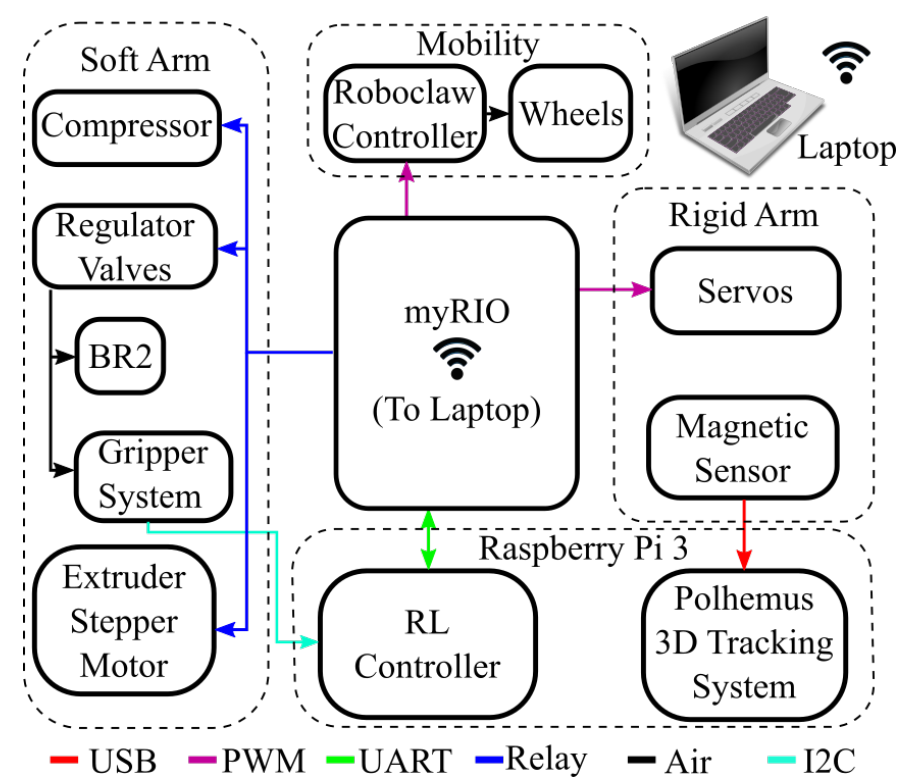

Fig. 4. A block diagram of the robot systems and control

controller, portable pneumatic compressor (Parker Hannifin, D1008-23-01, 50 psi maximum operating pressure, $12 \mathrm{~V}$ operating voltage), stepper motor and to the high torque servos (Servocity, CM-D950TW-400 Servo Gearbox). The overall communication of the robot system is shown in Figure 4 In addition, the myRIO also communicates with the Raspberry Pi 3 in order to facilitate the control of the soft arm which will be detailed in Section III. These components can be used to deploy the presented hybrid soft-rigid arm design on any field or mobile robot and can be used for myriad of applications like surveying, mining, disaster response to name a few other than the berry harvesting application focused on in this paper. To demonstrate the compatibility of the arm design with different mobile platforms, we successfully retrofitted the arm and the control components on TerraSentia [16], a commercially available agriculture robot platform (see Figure 1 .

\section{Sensing}

The distal end of the $\mathrm{BR}^{2}$ SCA can be appended with a modular end effector like a gripper (presented in [I-D), suction cup, or camera to name a few. However, to control the end effector of the arm, we need to accurately sense its end position. This is challenging as the SCA has complex deformation modes such as bending, twisting and spiralling (see Figure 3] [27]. Past research in soft robotics has demonstrated various concepts including resistive [7], inductive [8], liquid metals [19], and more recently embedded magnets [21] to sense the deformed shape. Most of these methods are limited to simplified deformations such as contraction, extension, and planar bending at relatively small length ranges $(<5 \mathrm{~cm})$. Techniques involving vision such as motion capture [1] cannot be easily transferred to a field environment, especially with the end of the arm occluded inside a bush. 


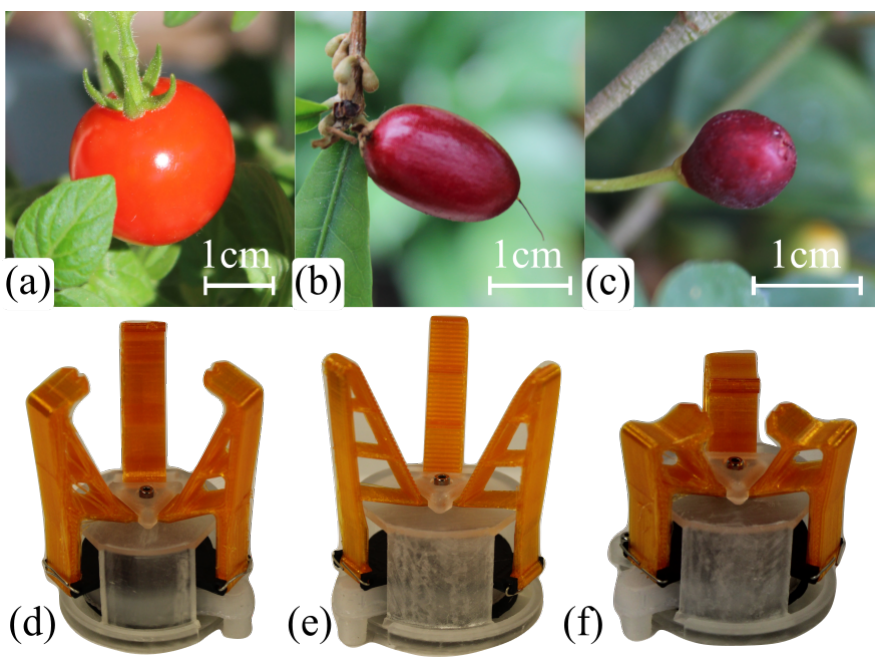

Fig. 5. Three fruits selected as the basis for gripper designs ((a) cherry tomato (b) miracle berry (c) small fig) (d)-(f) specialized grippers designed for each fruit. Gripper bases are $3.8 \mathrm{~cm}$ in diameter.

In this work, we use electromagnetic tracking (Patriot SEU, Polhemus) with a short range source (TX1, tracking area 2 to $60 \mathrm{~cm}$ ) and a micro flexible sensor as shown in Figure 2(d). This system is not constrained by a line of sight requirement. Furthermore, the sensor is flexible, lightweight $(<2 g)$ and does not hinder or alter the performance of the SCA. The electromagnetic source is mounted on the 3D-printed rigid enclosure and the sensor is inserted into a slot in the gripper base. The sensor has a positional accuracy of less than 1 $\mathrm{mm}$. The signal from the sensor provides the real time spatial coordinates of the soft arm end, which is then used for the soft continuum arm control (elaborated in Section III).

\section{Gripper system}

1) Gripper Design: We have designed three modular, pneumatically actuated, soft, compliant (3D-printed with TPU material) grippers as shown in Figure 5(d)-(f) to grasp three targeted fruits: Miracle berries, cherry tomatoes, and small figs (Figure 5(a)-(c)). These fruits were selected to explore a range of challenges related to mechanical harvesting. This pneumatically actuated gripper design was selected because of the limitations imposed by the soft arm. The gripper needed to be light weight due to the small payload of the arm. A gripper driven by servos and gears would significantly reduce the payload available for picking the fruit and add to the complexity of the design. A wire driven design was also explored, but due to the potential for interference with the performance of the soft arm, it was not selected. The pneumatic design also lent itself well to the existing equipment needed to control the soft arm.

The modular gripper system is attached to the soft arm via a simple mechanical catch and held in place with a screw. The air and sensor connections are accomplished via barbed nipples and DuPont style connectors respectfully.

The miracle berry is a firm fruit that sits close to the stem. As such, its gripper (Figure 5(d)) is designed to grip the

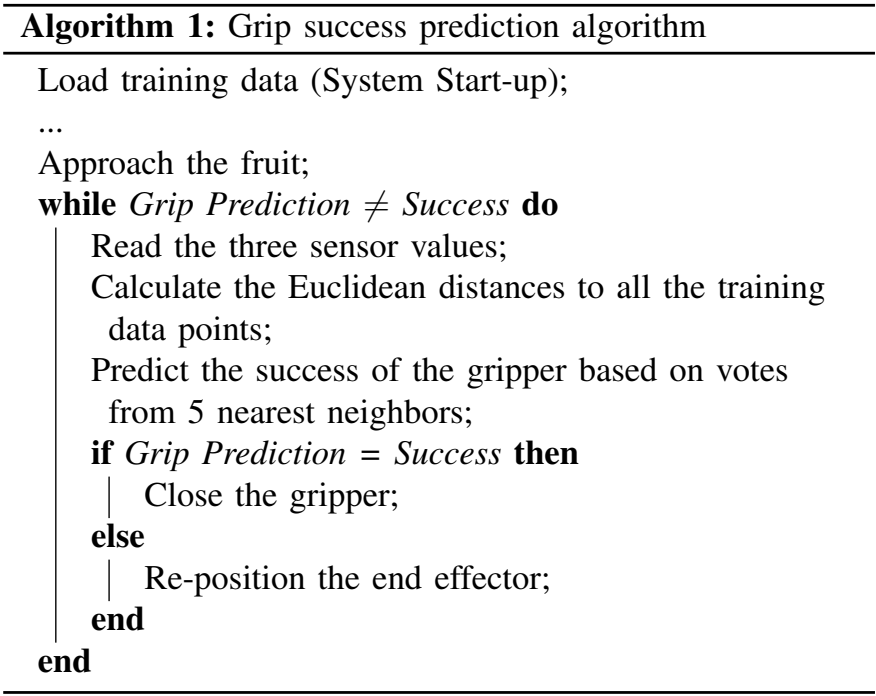

fruit directly. The fingers of this gripper were inspired by the FinGripper [9, 10] developed by Festo and are designed to conform to the shape of the fruit using the Fin Ray ${ }^{\circledR}$ effect [6] and evenly distribute the applied forces. By directly gripping the fruit, it is also possible to apply a twisting motion to aid in its removal. The fig is very soft and sits on a long stem. Direct gripping would damage the fruit, so instead the fig gripper (Figure 5(f)) was designed to enclose the fruit. Tabs on the gripper fingers close behind the fruit and when a pulling force is applied, it is directed axially along where the fig is attached to the stem. Once detached, it stays entrapped within the fingers until released into a storage container. The cherry tomato is also a soft fruit that sits on a stem, but like the miracle berry, it requires the application of a moment to detach it from the stem. As in the fig design, the gripper (Figure $5(\mathrm{~d})$ ) is designed to entrap the tomato and apply force behind the fruit. Because the fruit is soft, care must be taken when gripping it. The tabs prevent too much pressure from being applied and the finger's deformable shape evenly distributes the force over the surface of the fruit. This gripping style allows for a simultaneous pulling and twisting motion, which easily separates the tomato from the plant without damaging it.

2) Gripper Sensing: Operating within occluded environments, makes it challenging to determine if the end effector is positioned correctly to successfully grip a fruit. To overcome this and move toward autonomy, a sensing system has been incorporated into the gripper. The goal was to determine if the end effector is positioned correctly for a successful grip prior to closing the gripper. Previous work on object classification with a soft gripper has been done by Homberg et al. [14] and grasp success by Zimmer et al. [32]. These focused on tactile sensing and required contact with the fruit.

To predict success prior to contact, we used optical sensors in this work. Three IR reflectance sensors (Adafruit) were arranged around the edge of the gripper body, between the gripper fingers (Figure 2(c)). They are aimed to the center of 
the space between the gripper fingers where it is most likely for a body to be gripped successfully (Approximately $20 \mathrm{~mm}$ from the base of the fingers). The closer an object is to the sensor the higher the sensor output. To make a prediction from these three signals, Scikit-learn's k-nearest neighbor $(k \mathrm{NN})$ classifier [20] was used. Training was done with the gripper attached to a UR3 robotic arm (Universal Robots) to take advantage of the speed and precise positioning. To collect training data, a 3D-printed fruit was mounted on a flexible shaft and attached to the table surface. A random sample of the 400 points in and around the gripper was created and the gripper was moved so that the center of the fruit sits at one of the training points. The sensor readings were recorded, the gripper was closed and the success or failure state was logged manually. This data set is then used to predict the outcome of a grip attempt using a $k \mathrm{NN}$ classifier with $k=5$ as seen in Algorithm 11. This prediction is then sent to the operator to help them decide whether or not to grip or readjust the end effector position.

\section{CONTROL OF THE BR ${ }^{2}$ SCA AND TEleoperation}

\section{A. Learned Feedback Planning via Deep RL}

Given a desired reach point provided by a human operator, the $\mathrm{BR}^{2} \mathrm{SCA}$ system must choose a sequence of pressure change actions corresponding to bending and rotation $\left(\Delta P_{b}, \Delta P_{r}\right)$ of the continuum body to efficiently guide the end effector. Due to the highly nonlinear relationship between pressure changes and the end effector position, we would like to choose a general control strategy that may accommodate such a mapping and be executed in real-time. Rather than performing some system identification for specific arm settings and loading, we use a Kirchhoff rod model [2] of the soft arm to train a control policy directly from experience [24, 25]. Virtually any arm configuration and simulated loading can be trained using an existing reinforcement learning (RL) strategy called Deep Deterministic Policy Gradient (DDPG) introduced by Lillicrap et al. [18]. Our aim is to ultimately deploy the learned policy on the $\mathrm{BR}^{2} \mathrm{SCA}$ system, and hence we address the challenge of inevitable simulation model mismatch. We will show that defining an error state with respect to the end effector as an implicit feedback mechanism as well as operating in a quasi-static setting enables a useful policy in the real environment without further tuning.

1) Soft Arm MDP Formulation: Classically these problems are formulated as an infinite horizon discounted Markov decision process described by the tuple $\mathcal{M}=(\mathcal{S}, \mathcal{A}, \mathcal{P}, \mathcal{R}, \gamma)$ where the state transition probability $\mathcal{P}$ is unknown. We aim to find a policy $\pi$ which maximizes the expected discounted reward with trajectory distribution $\rho_{\pi}(\tau)$.

$$
\pi^{*}=\underset{\pi}{\arg \max } \mathbb{E}_{\tau \sim \rho_{\pi}(\tau)}\left[\sum_{t=0}^{\infty} \gamma^{t} \mathcal{R}\left(s_{t}, a_{t}\right)\right]
$$

We define the state $s \in \mathcal{S}$ by the current pressure states $\left(P_{b}, P_{r}\right)$, extensions length $L$ and the vector between the current measured end effector position $\hat{\mathbf{x}} \in \mathbb{R}^{3}$ and the desired position $\mathbf{x}_{d}$.

$$
s:=\left(\overline{\mathbf{x}}, P_{b}, P_{r}, L\right)
$$

Where $\overline{\mathbf{x}}=\mathbf{x}_{d}-\hat{\mathbf{x}}$. The policy chooses normalized pressure differences as actions $a:=\left(\Delta P_{b}, \Delta P_{r}\right) \in \mathcal{A}=[-1,1]^{2}$. The reward function $\mathcal{R}: \mathcal{S} \times \mathcal{S} \rightarrow \mathbb{R}$ is defined as in Satheeshbabu et al. [24] to achieve the desired end effector position in minimum time. We denote $\mathcal{S}_{T}$ as the set of terminal states (i.e. pressure constraints). This reward function provides incentive to make progress towards the goal at each step and achieve a successful reach in minimal time. Note that reward function arguments $\mathcal{R}\left(s_{t}, a_{t}\right)$ can implicitly represent arguments $\mathcal{R}\left(s_{t}, s_{t+1}\right)$.

$$
\mathcal{R}\left(s_{t}, s_{t+1}\right)= \begin{cases}-2+\left(\left\|\overline{\mathbf{x}}_{t}\right\|_{2}-\left\|\overline{\mathbf{x}}_{t+1}\right\|_{2}\right) & s_{t+1} \notin \mathcal{S}_{T} \\ -100-\left\|\overline{\mathbf{x}}_{t+1}\right\|_{2} & s_{t+1} \in \mathcal{S}_{T} \\ 100 & \left\|\overline{\mathbf{x}}_{t+1}\right\|_{2} \leq \epsilon\end{cases}
$$

Where $\epsilon$ defines a ball in which the end effector is sufficiently close to the target and is deemed successfully reached $(\epsilon=2$ $\mathrm{cm}$ in this case). There is a large penalty for exceeding the pressure constraints and not achieving a successful reach after each quasi-static pressure command with an additional penalty based on how far away the constraint violation occurred from the goal. Other reward functions may be considered such as ones that encourage energy efficiency using minimal pressure actuation. However, in the quasi-static setting achieving the goal in minimum number of steps is a reasonable heuristic for using minimum energy. A control policy can be learned based on this objective which we will now briefly describe.

2) DDPG Continuous Control Policy Optimization: With the objective of choosing actions that maximize the long term expected rewards through interacting with environment, it is useful to define an optimal value function conditioned on a particular action or a $Q$-function and can be written recursively with respect to a given policy $\pi$. We further assume that the current policy $\pi$ is a deterministic function of state as in Lillicrap et al. [18], where $\xi$ is some state distribution in the given MDP environment with reward map $\mathcal{R}$.

$$
Q^{\pi}\left(s_{t}, a_{t}\right)=\mathbb{E}_{\mathcal{R}, s_{t} \sim \xi}\left[\mathcal{R}\left(s_{t}, a_{t}\right)+\gamma Q^{\pi}\left(s_{t+1}, \pi\left(s_{t+1}\right)\right)\right]
$$

In Deep Deterministic Policy Gradient (DDPG), the Qfunction is parameterized as a fully-connected neural network, $Q_{\theta}$, with parameters $\theta$. It also utilizes an actor-critic architecture with actor network $\pi_{\phi}$ and parameters $\phi$ which will serve as the control policy. The recursive definition of thr Q-function yields the following loss function given the current Q-function and observed reward $r$ at time $t$ used to optimize $Q_{\theta}$.

$$
\mathcal{L}\left(\theta_{i}\right)=[\underbrace{r_{t}+\gamma Q_{\theta_{i-1}}\left(s_{t+1}, \pi_{\phi}\left(s_{t+1}\right)\right)}_{\text {target }}-Q_{\theta_{i}}\left(s_{t}, a_{t}\right)]^{2}
$$

DDPG uses the previous network parameters as a loss target, and then performs a weighted "soft update" to the target Qfunction parameters. The policy gradient for updating the actor network $\pi_{\phi}$ may be computed using the critic $Q_{\theta}$, all of which is described in detail in Lillicrap et al. [18]. DDPG enjoys the benefits of being off-policy meaning that the policy may be updated even from state-action trajectories from another policy (e.g. a random policy), allowing stable learning by sampling 


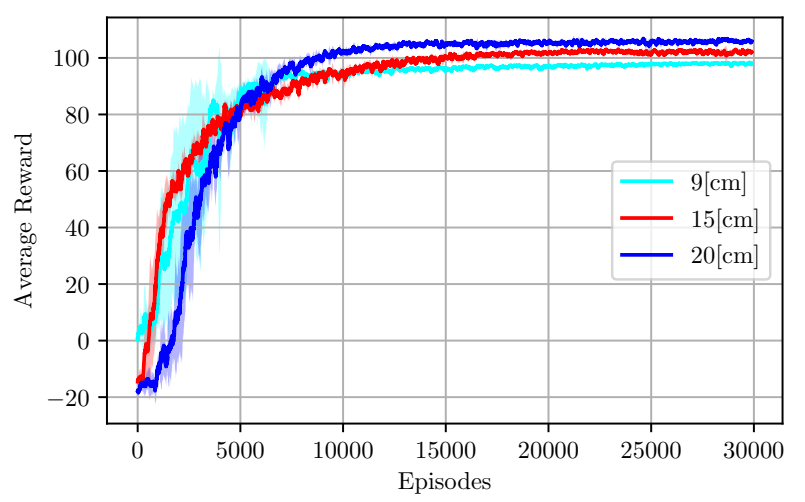

Fig. 6. 100-reward average training curves are displayed over $30 \mathrm{~K}$ reach episodes for lengths 9,15 and $20 \mathrm{~cm}$ of the extruded soft continuum arm. These curves are an average over 5 independent complete training trials from random initial policy networks. The shaded regions denote the variance over each these training trials at each length.

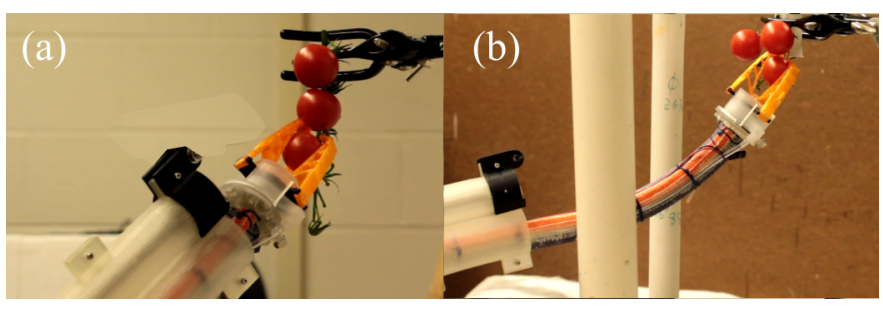

Fig. 7. Picking cherry tomatoes (a) with soft continuum arm retracted and (b) with extruded soft continuum arm in the presence of obstacles in the path. Cherry tomatoes are approximately $2.5 \mathrm{~cm}$ in diameter.

from a large experience buffer. As shown in Figure 6, training from simulation of various physical configurations are stable and consistent.

\section{B. Control and Teleoperation}

The rigid arm is controlled in task space by user supplied $\Delta X, \Delta Y$, and $\Delta Z$. This is accomplished through analytically derived inverse kinematics [26].

The soft arm is also controlled in the task space by user supplied $\Delta x, \Delta y$, and $\Delta z$. This is accomplished via the RL policy described above. The user also controls $\Delta L$ (extrusion length) and $\Delta \theta_{4}$ (axial rotation of the VaLeNS arm).

\section{VALIDATION AND FIELD TRIALS}

\section{A. Validation of gripper sensing system}

The gripper sensing system was validated both prior to implementation into the system and after. Prior to implementing, the training data set was validated by testing it on a UR3 arm (Universal Robots). Using 80 test points, it was able to achieve $88 \%$ precision in predicting success correctly and $100 \%$ precision in predicting failure correctly. As seen in Figure 8, the distinction between success and failure sensing output is strong and lends itself to robust results.

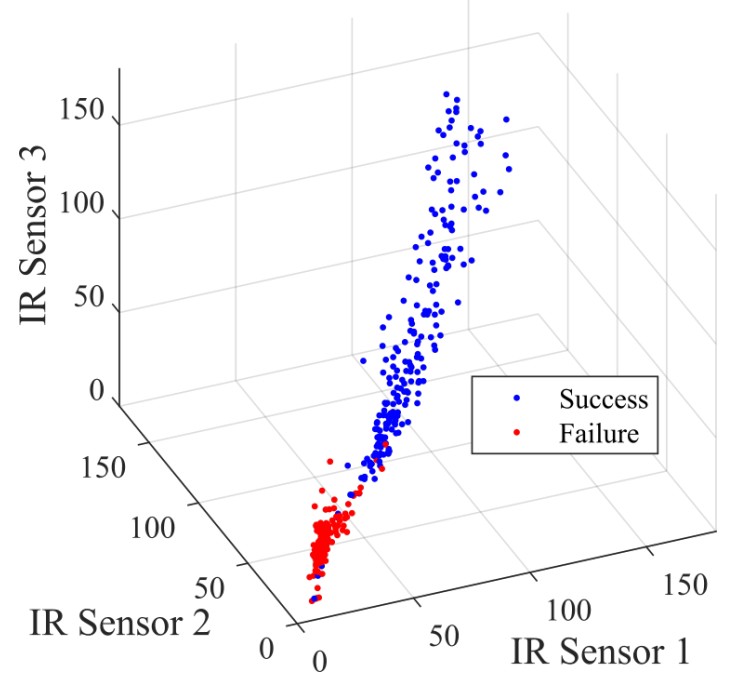

Fig. 8. A 3D representation of gripper success as a function of the IR sensor readings based on the training data showing the separation of the success and failure.

\section{B. Picking maneuvers}

There are two main tasks in order to successfully pick a berry. First, the gripper has to reach the berry successfully and grip it softly. Second, it should be able to sever the berry from the branch. Although these tasks appear trivial when done by humans, it involves a combination of dexterous hand motions. While past research on rigid link robots have tackled this problem, the most notable being a blade that attaches to the gripper to shear the branch by Xiong et al. [31], these strategies may not be compatible for a soft manipulator. In this paper we will explore severing the berries by multiple maneuvers involving griping and pulling. It is to be noted that once the gripper successfully grips the berry, the grip remains intact even after removing the air pressure in SCA. Here we list down the different feasible maneuvers with the current arm design. Each of them are tested after the gripper senses the berry.

- SCA retraction: Where the SCA is retracted by $7 \mathrm{~cm}$ as shown in Figure 3(a) represented by $\Delta L$.

- Rigid link retraction: In this maneuver, the rigid arm is retracted backwards from the plant. It maps to a change in the $\theta_{2}$ angle in Figure 3(a) marked as 2.

- Hybrid arm twist: The $\theta_{4}$ angle (in Figure 3 (a) marked as 4) which corresponds to an axial rotation of the VaLeNS arm by 60 degrees.

- Sideward flick: In this maneuver, the rigid arm is moved in a sidewards direction ( $\mathrm{x}$ or $\mathrm{y}$ axis movement) which corresponds to a change in the $\theta_{1}$ angle shown in Figure 3 (a) marked as 5.

- Downward flick: Similar to the sideward flick, the rigid arm is moved downwards (-z direction) which corresponds to change in the $\theta_{3}$ angle shown in Figure 3 (a) marked as 3.

- SCA twist: The rotating actuators of $\mathrm{BR}^{2} \mathrm{SCA}$ are 
actuated in order to obtain the twist motion of the $\mathrm{BR}^{2}$ SCA.

The efficacy of each of these picking maneuvers is evaluated in two different scenarios. In the first scenario, there is no obstacle in between the berry and the approach path of the arm. In the second scenario, an obstacle (as shown in Figure 7(b)) is placed in the path of the arm. In each scenario, the berry is securely gripped by the gripper and the above maneuvers are implemented in order to see the efficacy of the picking maneuvers. Furthermore, these experiments are conducted under two sub cases. The first sub case is with the SCA fully retracted (as shown in Figure 7(a)), which corresponds to the case when berries are picked from the periphery. The second sub case is when the extruded length is greater than $7 \mathrm{~cm}$ $(10 \mathrm{~cm}, 15 \mathrm{~cm}$ and $20 \mathrm{~cm}$ ) (as shown in 7b)) to include the scenario when the berries are inside the bush. Table II and Table III shows the success of different maneuvers for each sub case. It can be observed from the results that retracting the SCA works for all the tested lengths except when there is zero extrusion. Retraction using rigid links, sideward flick and downward flick are also successful for most of the cases. However these are contingent on the surrounding environment and possible damage caused to neighboring berries. From Table $[\mathrm{I}$ and Table $\mathrm{II}$ it is also evident that twist using hybrid arm and twist using SCA rotation are successful in only a few cases. The berry was successfully picked only when the SCA and hybrid arm rotation are repeated 3 times on an average.

TABLE I

SCENARIO 1: PicKing MANEUVERS WITH NO OBSTACLE

\begin{tabular}{ccccc} 
& \multicolumn{5}{c}{ Extruded Length of the SCA } \\
\hline Maneuver & $\mathbf{0 ~ c m}$ & $\mathbf{1 0}$ cm & $\mathbf{1 5}$ cm & $\mathbf{2 0} \mathbf{~ c m}$ \\
\hline SCA retraction & X & $\checkmark$ & $\checkmark$ & $\checkmark$ \\
Rigid link retraction & $\checkmark$ & $\checkmark$ & $\checkmark$ & $\checkmark$ \\
SCA twist & $\checkmark^{*}$ & $\checkmark^{*}$ & $\checkmark^{*}$ & $\checkmark$ \\
Hybrid arm twist & $\checkmark$ & x & x & $\checkmark$ \\
Sideward flick & $\checkmark$ & $\checkmark$ & $\checkmark$ & $\checkmark$ \\
Downward flick & $\checkmark$ & $\checkmark$ & $\checkmark$ & $\checkmark$ \\
\hline * more than three rotations & $\checkmark$ successful & x unsuccessful & &
\end{tabular}

TABLE II

SCENARIO 2: Picking MANEUVERS WITH SINGLE ObSTAClE

Extruded Length of the SCA

\begin{tabular}{ccccc}
\hline Maneuver & $\mathbf{0 ~ c m}$ & $\mathbf{1 0} \mathbf{~ c m}$ & $\mathbf{1 5} \mathbf{~ c m}$ & $\mathbf{2 0} \mathbf{~ c m}$ \\
\hline SCA retraction & X & $\checkmark$ & $\checkmark$ & $\checkmark$ \\
Rigid link retraction & x & $\checkmark$ & $\checkmark$ & $\checkmark$ \\
SCA twist & x & $\checkmark^{*}$ & $\checkmark^{*}$ & x \\
Hybrid arm twist & x & x & x & $\checkmark$ \\
Sideward flick & x & $\checkmark^{+}$ & $\checkmark^{+}$ & $\checkmark^{+}$ \\
Downward flick & x & $\checkmark^{+}$ & $\checkmark^{+}$ & $\checkmark^{+}$ \\
\hline + setup dependent & $\checkmark$ successful & x unsuccessful & &
\end{tabular}

\section{Soft arm control}

In section III we described a DDPG planning and control algorithm that is trained from a simulated Kirchhoff rod model of the soft arm. Although the control operates in a quasi-static setting, it is expected for the true SCA mechanics and actuation to differ from that of the simulation. To evaluate the controller

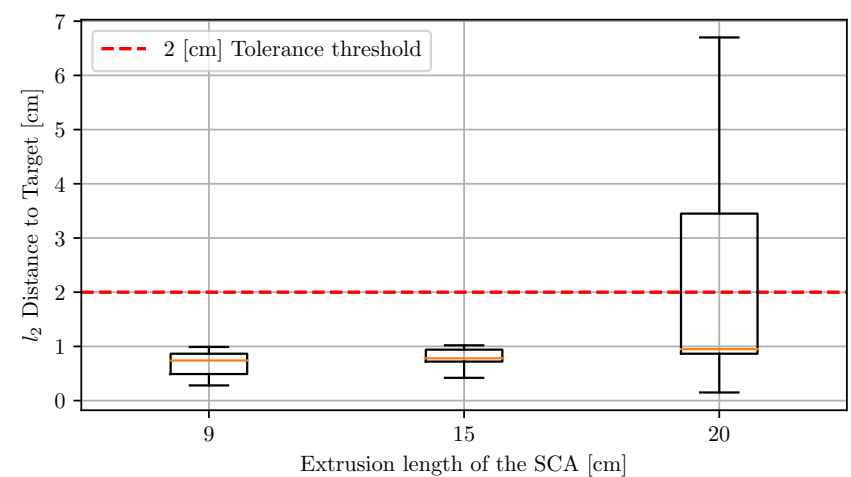

Fig. 9. In order to evaluate the DDPG policy on the $\mathrm{BR}^{2} \mathrm{SCA}$ system, for each length we select 15 reach points in the workspace when the arm is in the neutral position. For each point the arm is given 10 steps to reach the goal and stops once the error becomes less than $1 \mathrm{~cm}$. In practice $2 \mathrm{~cm}$ tolerance is acceptable. The orange line indicate the median final error distance which is under the desired threshold for all extension lengths.

we examine the ability of the $\mathrm{BR}^{2} \mathrm{SCA}$ to reach various points in the feasible workspace at 3 extrusion lengths $(9,15,20$ $[\mathrm{cm}])$ keeping the rigid arm position fixed. The points are chosen by discretizing the the bending and rotating pressure value $\left(P_{b}, P_{r}\right)$, setting the SCA to those pressures and then recording the delta $\overline{\mathbf{x}}$ via the electromagnetic sensor. Then, given the same initial conditions (i.e. position and pressures), we provide the DDPG algorithm the prescribed delta. Then one of the three following cases occurs:

1) the error to the point exceeds a 1 centimeter lower tolerance so that $\|\overline{\mathbf{x}}\|_{2}<1$ [cm]. Control terminates and the reach is deemed successful.

2) After 10 controller steps the final error is within the upper 2 centimeter tolerance so that $1 \leq\|\overline{\mathbf{x}}\|_{2} \leq 2$ [cm] and the reach is deemed successful.

3) After 10 controller steps the final error is $\|\overline{\mathbf{x}}\|_{2} \geq 2$ $[\mathrm{cm}]$ and the reach is considered a failure.

Then the final error is recorded. We choose 15 points for each length and disregard pressure configurations that purely rotate the SCA, as the resulting delta is very small and not meaningful. For each $P_{b}$ state of $(20,30,40)$ psi we consider the rotation pressures $P_{r}$ states $(-40,-20,0,20,30,40)$ psi giving us the 15 corresponding workspace points at a given length. In Figure 9 we display the box plots of those reach trials. We note that for length 9 and 15 centimeters, every reach point achieved below the desired 2 centimeter threshold. However for 20 centimeters extension, 4/15 points exceeded the threshold, 3 of which points were at high bending pressures (30 psi or $40 \mathrm{psi}$ ). We suspect that for the longest length of 20 centimeters, the model mismatch of simulation and the real $\mathrm{BR}^{2} \mathrm{SCA}$ system are emphasized. A possible way to mitigate these failed reaches is to fine tune the DDPG controller on additional additional real reach experiences. 

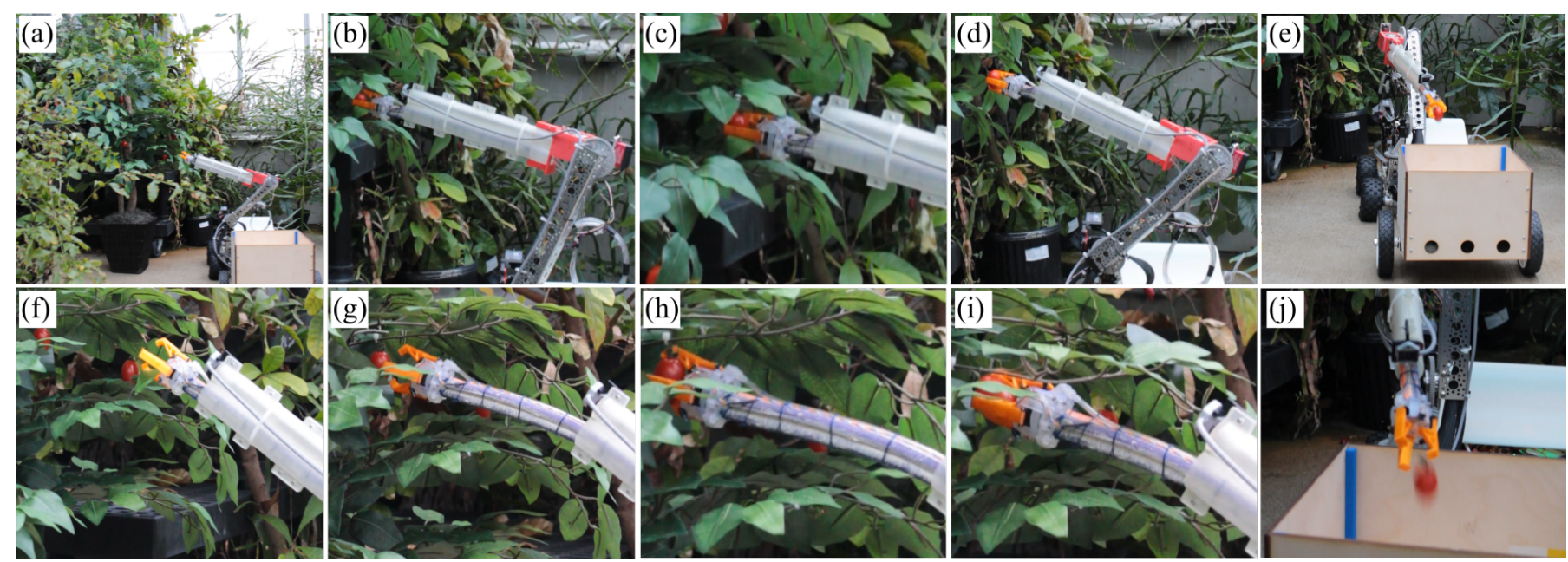

Fig. 10. System validation trial: (a) approaching the plant (b) reaching for a fruit on the periphery (no soft arm extrusion) (c) grasping the fruit (d) picking the fruit with rigid arm flick maneuver (e) depositing the fruit in a collection bin (f) reaching for a fruit in the interior of the plant (g) using soft continuum arm (SCA) extrusion to reach the fruit (h) grasping the fruit (i) picking the fruit with SCA retraction maneuver and (j) depositing the fruit in a collection bin.

\section{System validation}

In order to validate the entire system, the robot is controlled by the user to pick berries on the periphery and at different interior depths of the plant. Cherry tomatoes on a vine are attached to the branches of the plant in different locations. Figure 10 shows the different steps in successfully picking the berries. Figure 10 (a)-(e) show picking a berry from the periphery of the plant whereas Figure 10(f)-(h) show picking a berry from the internal depth of the plant. It can be observed in Figure 10(b)-(d) that in the case where berries are picked from the periphery of the plant, the task is performed with almost negligible extrusion of the SCA. Whereas, for the case when the berry is inside the bush, the SCA is extruded to reach the berry and to ensure soft interaction with the plant. In Figure 10 (c) and (h) it is shown how the gripper successfully grips the berry. In Figure 10(d) and (i) rigid arm retraction and soft arm extrusion maneuvers are used respectively to sever the berry from the branch and finally place it in the cart attached to the robot in Figure 10(e) and (j). The video of the robot performing several tasks is included in a supplementary video file 1

\section{E. Discussion}

It is observed that the picking of peripheral berries was straightforward, relatively simple and efficient with the task space control presented in this work. This is because peripheral berries required actuation of the rigid links alone. Although this system possesses the required capabilities to successfully pick berries from the inside of a bush, the current task space control renders this operation to be slow. For example, a berry located $15 \mathrm{~cm}$ inside the bush required approximately 4 minutes to reach, grip and pick. This required 12 user commands (5 for the rigid arm and 7 for the soft arm) to accomplish. The two main challenges observed involve the

\footnotetext{
${ }^{1}$ https://youtu.be/0hFTPOUUaIE
}

issue of perception and convergence of SCA control. The issue of perception is the difficulty to visually perceive the location of the berry by the user. The IR reflectance sensor on the gripper aided in overcoming perception challenges, but is only effective during the final stages of approach towards the berry. Furthermore, this sensor did experience some false positive readings when chunks of leaves rather than a berry entered the gripping space.

\section{FUTURE WORK AND CONCLUSIONS}

We have presented a hybrid rigid-soft arm and manipulator for performing tasks requiring dexterity and reach in cluttered environments. We validated the system in the challenging agricultural problem of picking berries. The system however can be modified for use in other applications requiring dexterity and reach. Future work can include improving gripper sensing and utilizing it in the control loop to fine tune to positioning of the gripper and achieve better gripping results. The training can also be made more robust by training it in the full environment and developing the ability to differentiate between target fruits and other materials such as branches and leaves. Our system as presented uses reinforcement learning to track desired task-space trajectories by controlling pneumatic pressures in the arm. This makes the system easy for a human to operate, but it is not yet fully autonomous. However, the system will allow users to demonstrate feasible trajectories for the future development of a fully autonomous berry picking systems.

\section{ACKNOWLEDGMENTS}

This work is jointly funded by NIFA through the NIFANSF National Robotics Initiative USDA 2019-67021-28989. In addition, the authors would like to acknowledge Marc Abraldes Velasco and Sri Theja for their support in building the robot and recording the video. 


\section{REFERENCES}

[1] Yasmin Ansari, Mariangela Manti, Egidio Falotico, Yoan Mollard, Matteo Cianchetti, and Cecilia Laschi. Towards the development of a soft manipulator as an assistive robot for personal care of elderly people. International Journal of Advanced Robotic Systems, 14 (2):172988141668713, 4 2017. ISSN 1729-8814. doi: $10.1177 / 1729881416687132$.

[2] Stuart S Antman. Nonlinear Problems of Elasticity, 1995, 1995.

[3] Yin Bao, Lie Tang, Matthew W. Breitzman, Maria G. Salas Fernandez, and Patrick S. Schnable. Field-based robotic phenotyping of sorghum plant architecture using stereo vision. Journal of Field Robotics, 36(2):397-415, 3 2019. ISSN 15564959. doi: 10.1002/rob.21830. URL http://doi.wiley.com/10.1002/rob.21830.

[4] Adinor Jos Capellesso, Ademir Antonio Cazella, Abdon Luiz Schmitt Filho, Joshua Farley, and Diego Albino Martins. Economic and environmental impacts of production intensification in agriculture: comparing transgenic, conventional, and agroecological maize crops. Agroecology and Sustainable Food Systems, 40(3):215236, 3 2016. ISSN 2168-3565. doi: 10.1080/21683565. 2015.1128508 .

[5] Girish Chowdhary, Mattia Gazzola, Girish Krishnan, Chinmay Soman, and Sarah Lovell. Soft robotics as an enabling technology for agroforestry practice and research. Sustainability, 11(23):6751, 2019.

[6] Whitney Crooks, Gabrielle Vukasin, Maeve OSullivan,

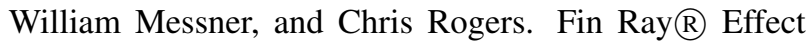
Inspired Soft Robotic Gripper: From the RoboSoft Grand Challenge toward Optimization. Frontiers in Robotics and AI, 3, 11 2016. ISSN 2296-9144. doi: 10.3389/ frobt.2016.00070.

[7] Wyatt Felt, Khai Yi Chin, and C David Remy. Contraction Sensing with Smart Braid McKibben Muscles. IEEE/ASME Transactions on Mechatronics, 21(3):12011209, 2016. ISSN 10834435. doi: 10.1109/TMECH. 2015.2493782.

[8] Wyatt Felt, Maria J. Telleria, Thomas F. Allen, Gabriel Hein, Jonathan B. Pompa, Kevin Albert, and C. David Remy. An inductance-based sensing system for bellowsdriven continuum joints in soft robots. Autonomous Robots, 43(2):435-448, 2 2019. ISSN 0929-5593. doi: 10.1007/s10514-018-9769-7.

[9] Festo. BionicTripod with FinGripper, 2009.

[10] Festo. Getting to Grips with the Adaptive Gripper DHDG, 2011. URL https://www.festo.com/net/sv_se/ SupportPortal/Details/210356/PressArticle.aspx

[11] Mario M. Foglia and Giulio Reina. Agricultural robot for radicchio harvesting. Journal of Field Robotics, 23 (6-7):363-377, 6 2006. ISSN 1556-4959. doi: 10.1002/ rob.20131. URL http://doi.wiley.com/10.1002/rob.20131.

[12] Jonathan A. Foley, Navin Ramankutty, Kate A. Brauman, Emily S. Cassidy, James S. Gerber, Matt Johnston,
Nathaniel D. Mueller, Christine OConnell, Deepak K. Ray, Paul C. West, Christian Balzer, Elena M. Bennett, Stephen R. Carpenter, Jason Hill, Chad Monfreda, Stephen Polasky, Johan Rockström, John Sheehan, Stefan Siebert, David Tilman, and David P. M. Zaks. Solutions for a cultivated planet. Nature, 478(7369):337-342, 10 2011. ISSN 0028-0836. doi: 10.1038/nature10452.

[13] H. Charles J. Godfray and Tara Garnett. Food security and sustainable intensification. Philosophical Transactions of the Royal Society B: Biological Sciences, 369(1639):20120273, 4 2014. ISSN 0962-8436. doi: 10.1098/rstb.2012.0273.

[14] Bianca S. Homberg, Robert K. Katzschmann, Mehmet R. Dogar, and Daniela Rus. Robust proprioceptive grasping with a soft robot hand. Autonomous Robots, 43(3): 681-696, 3 2019. ISSN 0929-5593. doi: 10.1007/ s10514-018-9754-1.

[15] Pl Johan From, Lars Grimstad, Marc Hanheide, Simon Pearson, and Grzegorz Cielniak. RASberry - Robotic and Autonomous Systems for Berry Production. Mechanical Engineering, 140(06):S14-S18, 62018. ISSN 0025-6501. doi: 10.1115/1.2018-JUN-6. URL https://asmedigitalcollection.asme.org/ memagazineselect/article/140/06/S14/447707/ RASberry-Robotic-and-Autonomous-Systems-for-Berry

[16] Erkan Kayacan, Zhongzhong Zhang, and Girish Chowdhary. Embedded high precision control and corn stand counting algorithms for an ultra-compact $3 \mathrm{~d}$ printed field robot. Proceedings of Robotics: Science and Systems. Pittsburgh, Pennsylvania, 2018.

[17] Cecilia Laschi and Matteo Cianchetti. Soft Robotics: New Perspectives for Robot Bodyware and Control. Frontiers in Bioengineering and Biotechnology, 2:3, 2014. ISSN 2296-4185. doi: 10.3389/fbioe.2014. 00003. URL http://journal.frontiersin.org/article/10. 3389/fbioe.2014.00003.

[18] Timothy P. Lillicrap, Jonathan J. Hunt, Alexander Pritzel, Nicolas Heess, Tom Erez, Yuval Tassa, David Silver, and Daan Wierstra. Continuous control with deep reinforcement learning. arXiv preprint arXiv:1509.02971, 92015.

[19] Yong Lae Park and Robert J Wood. Smart pneumatic artificial muscle actuator with embedded microfluidic sensing. Proceedings of IEEE Sensors, pages 1-4, 2013. ISSN 1930-0395. doi: 10.1109/ICSENS.2013.6688298.

[20] Fabian Pedregosa, Gal Varoquaux, Alexandre Gramfort, Vincent Michel, Bertrand Thirion, Olivier Grisel, Mathieu Blondel, Peter Prettenhofer, Ron Weiss, Vincent Dubourg, Jake Vanderplas, Alexandre Passos, David Cournapeau, Matthieu Brucher, Matthieu Perrot, and douard Duchesnay. Scikit-learn: Machine Learning in Python. Journal of Machine Learning Research, 12: 2825-2830, 2011.

[21] Panagiotis Polygerinos, Nikolaus Correll, Stephen A. Morin, Bobak Mosadegh, Cagdas D. Onal, Kirstin Petersen, Matteo Cianchetti, Michael T. Tolley, and Robert F. Shepherd. Soft Robotics: Review of Fluid- 
Driven Intrinsically Soft Devices; Manufacturing, Sensing, Control, and Applications in Human-Robot Interaction. Advanced Engineering Materials, 19(12): 1700016, 12 2017. ISSN 14381656. doi: 10.1002/adem. 201700016.

[22] Daniela Rus and Michael T Tolley. Design, fabrication and control of soft robots. Nature, 521(7553):467-475, 2015.

[23] Jayesh B. Samtani, Curt R. Rom, Heather Friedrich, Steven A. Fennimore, Chad E. Finn, Andrew Petran, Russell W. Wallace, Marvin P. Pritts, Gina Fernandez, Carlene A. Chase, Chieri Kubota, and Brad Bergefurd. The Status and Future of the Strawberry Industry in the United States. HortTechnology, 29(1):11-24, 22019. ISSN 1063-0198. doi: 10.21273/HORTTECH04135-18. URL https://journals.ashs.org/view/journals/horttech/29/ 1/article-p11.xml.

[24] Sreeshankar Satheeshbabu, Naveen Kumar Uppalapati, Girish Chowdhary, and Girish Krishnan. Open Loop Position Control of Soft Continuum Arm Using Deep Reinforcement Learning. In 2019 International Conference on Robotics and Automation (ICRA), pages 5133-5139, Montreal, 5 2019. IEEE. ISBN 978-1-5386-6027-0. doi: 10.1109/ICRA.2019.8793653.

[25] Sreeshankar Satheeshbabu, Naveen K Uppalapati, Tianshi Fu, and Girish Krishnan. Continuous Control of a Soft Continuum Arm using Deep Reinforcement Learning. In IEEE International Conference on Soft Robotics, 2020.

[26] Mark W. Spong, Seth Hutchinson, and M. Vidyasagar. Robot modeling and control. IEEE Control Systems, 26 (6):113-115, 2006. ISSN 1066033X. doi: 10.1109/MCS. 2006.252815 .

[27] Naveen K Uppalapati and Girish Krishnan. Design of Soft Continuum Manipulators Using Parallel Asymmetric Combination of Fiber Reinforced Elastomers, 82018.

[28] Naveen Kumar Uppalapati and Girish Krishnan. Towards Pneumatic Spiral Grippers: Modeling and Design Considerations. Soft Robotics, page soro.2017.0144, 72018. ISSN 2169-5172. doi: 10.1089/soro.2017.0144.

[29] Naveen Kumar Uppalapati and Girish Krishnan. VaLeNS: Design of a Novel Variable Length Nested Soft Arm. IEEE Robotics and Automation Letters, 5(2):11351142, 4 2020. ISSN 2377-3766. doi: 10.1109/LRA.2020. 2967303.

[30] Naveen Kumar Uppalapati, Gaurav Singh, and Girish Krishnan. Parameter estimation and modeling of a pneumatic continuum manipulator with asymmetric building blocks. In 2018 IEEE International Conference on Soft Robotics (RoboSoft), pages 528-533. IEEE, 42018. ISBN 978-1-5386-4516-1. doi: 10.1109/ROBOSOFT. 2018.8405380.

[31] Ya Xiong, Cheng Peng, Lars Grimstad, Pl Johan From, and Volkan Isler. Development and field evaluation of a strawberry harvesting robot with a cable-driven gripper. Computers and Electronics in Agriculture, 157:392-402,
2 2019. ISSN 01681699. doi: 10.1016/j.compag.2019. 01.009 .

[32] Julian Zimmer, Tess Hellebrekers, Tamim Asfour, Carmel Majidi, and Oliver Kroemer. Predicting Grasp Success with a Soft Sensing Skin and Shape-Memory Actuated Gripper. In Proceedings of (IROS) IEEE/RSJ International Conference on Intelligent Robots and Systems, pages 7120-7127, 2019. ISBN 9781728140032. 